\title{
Antenna-Coupled Light Emission from Two-Dimensional Materials
}

\author{
Palash Bharadwaj*a , Markus Parzefall ${ }^{\mathrm{b}}$, Achint Jain ${ }^{\mathrm{b}}$, Lukas Novotny ${ }^{\mathrm{b}}$
}

${ }^{a}$ Electrical and Computer Engineering, Rice University, Houston, TX 77005

b Photonics Laboratory, ETH Zurich, 8093 Zurich, Switzerland

\begin{abstract}
We have studied the ultrafast conversion of electrons localized in vertical Au-hBN-Au tunnel junctions to free-space photons, mediated by resonant slot antennas. We achieve polarized, directional and resonantly enhanced light emission from inelastic electron tunneling and establish a novel platform for studying the interaction of electrons with strong electromagnetic fields. Further, we show that plasmonic antennas made from a gold nanoparticle dimer coupled with a semiconducting $2 \mathrm{D}$ material $\left(\mathrm{MoS}_{2}\right)$ offer control over emission directionality.
\end{abstract}

Keywords: Optical antennas, tunnel junctions, two dimensional materials.

\section{INTRODUCTION}

Transition metal dichalcogenide (TMDC) monolayers are exciting for optoelectronic applications because of their direct bandgap in the visible-near IR part of the spectrum. Boron nitride (h-BN), a large bandgap insulator, complements semiconducting TMDCs and graphene. A variety of devices such as LEDs and photodetectors using TMDC monolayers have recently been demonstrated ${ }^{1,2}$. On the other hand, plasmonics has established itself over the last decade as a viable means to control light-matter interaction in confined nanoscopic volumes. Optical antennas are elements that couple free space radiation to nanoscale volumes. To date, optical antennas have been driven using light, in contrast to their radio frequency counterparts which are driven by electrical currents. We have realized ultrafast solid-state light-emitting tunnel devices based on arrays of electrically driven optical antennas that significantly alter this paradigm.

To achieve this electro-optical transduction, we use vertical Au-hBN-Au tunnel junctions that exhibit remarkable stability compared to other tunnel junctions studied thus far under high biases ${ }^{3}$. Electrons tunnel from one electrode to the other under an applied bias, and generate highly-confined surface plasmons by inelastic tunneling. In earlier works, we found that inelastic electron tunneling from the tip of an ambient scanning tunneling microscope (STM) to a gold film or gold nanowire can excite propagating surface plasmons on the gold-air interface ${ }^{4}$. This process has been known since the pioneering work of Lambe and McMarthy who found that surface plasmon modes in metal-insulator-metal (MIM) junction increase the probability of inelastic tunneling, and that these surface plasmons may subsequently decay into farfield radiation. This effect has since been studied experimentally in macroscopic solid-state tunnel junctions ${ }^{5}$, and the scanning tunneling microscope ${ }^{6}$. One major appeal of investigating inelastic electron tunneling is its potential speed. Because it does not rely on intermediate excitations such as electron-hole pairs, the response time of such devices is fundamentally only limited by the tunneling time of electrons through the junction, a process that takes place on a femtosecond timescale. This enables tunnel devices to operate at optical frequencies.

The tunnel barrier of top-down fabricated devices is commonly based on oxides grown on one of the electrodes (for example, aluminium oxide) or on insulating films deposited by atomic layer deposition or sputtering. Unfortunately, due to grain formation and defects, these tunnel barriers are unstable, and operation over an extended time at ambient conditions is not possible. Here, we investigate light generation via inelastic electron tunneling in microscopic tunnel devices comprised of gold ( $\mathrm{Au}$ ) and hexagonal boron nitride (h-BN). h-BN provides a stable, high-quality tunnel barrier due to its crystallinity and its large bandgap of $\sim 6 \mathrm{eV}$. We find that light emission can be enhanced and spectrally controlled by nanostructuring one of the electrodes. The light emission and optical properties of the device are found to be closely related. Furthermore, we demonstrate modulation of the emitted light at frequencies up to $1 \mathrm{GHz}$. 


\section{RESULTS AND DISCUSSION}

\subsection{Light emission from h-BN tunnel junctions}

Figure 1 shows the sample configuration. A circular Au bottom electrode $(1 \mathrm{~nm} \mathrm{Ti} / 50 \mathrm{~nm} \mathrm{Au})$ is photolithographically defined, then nanostructured by focused ion beam milling (FIB). The bottom electrode is segmented into four quartercircle electrodes, which are wired individually as shown in Figure 1B. One electrode remains unstructured to serve as a reference, while the remaining three electrodes are structured into arrays of optical antennas in the form of rectangular slots. The distance between slots is kept constant at $\sim 100 \mathrm{~nm}$ along both the long and short sides of the rectangles, and the nominal size of the slots is $150 \times 50,250 \times 50$ and $350 \times 50 \mathrm{~nm}^{2}$ for devices 1,2 and 3, respectively. Exfoliated fewlayer h-BN (here, nine atomic layers, or $\sim 3 \mathrm{~nm}$ ) is transferred on top of the bottom electrode. The device is finished by the fabrication of a common Au top electrode (15 nm Au) via photolithography.
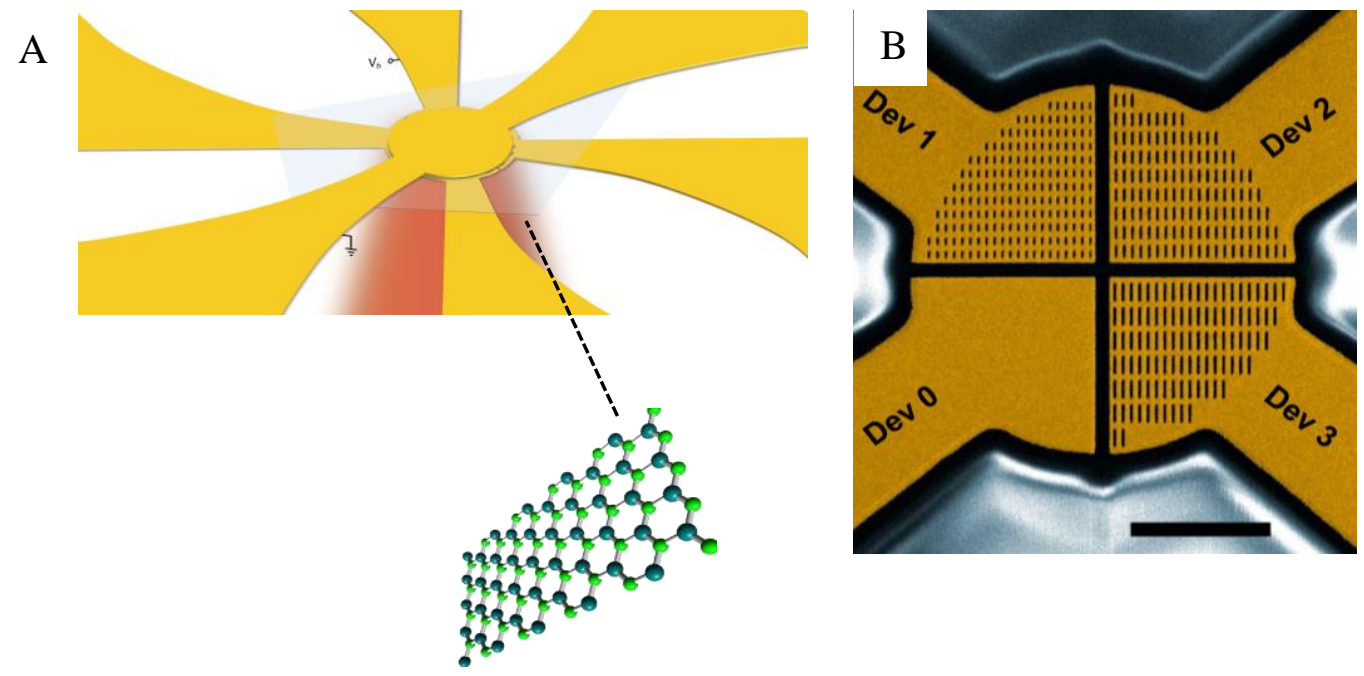

Figure 1. (A) Schematic of the fabricated device comprising two gold electrodes and a thin flake of $\mathrm{hBN}$ between them. (B) A false color scanning electron micrograph of the bottom electrode before transferring the hBN. The slot antennas in different sectors are different aspect ratios and hence varying spectral resonances. The scale bar is 1 micron.

The transmission of light through the devices strongly depends on the (linear) light polarization. Observing light polarized along the short axis of the slots results in a strong color contrast between devices. This contrast vanishes when observing light polarized along the long axis. The colors of the electrodes suggest that the slots enhance transmission in a certain spectral range. All structured devices exhibit characteristic resonances and enhanced transmission at wavelengths corresponding to the observed colors. The transmission peak shifts towards longer wavelengths as the aspect ratio of the slots is increased.

Next, we discuss the properties of photon emission induced by inelastic electron tunneling. When the unstructured reference device is biased at a constant tunnel voltage of $V_{\mathrm{b}}=2.5 \mathrm{~V}$, light emission is found to be localized to the edges of the device. The spectrum of the emitted light, is broad and without distinct features, falling off continuously towards shorter wavelengths. In the absence of slots, as is the case for the reference device, inelastically tunneling electrons predominantly interact with SPPs associated with the MIM configuration ${ }^{7,8}$. In the absence of discontinuities, these modes cannot couple to free-space radiation because of the large momentum mismatch. However, scattering due to surface roughness or edges provides the momentum required to overcome this mismatch.

In contrast, all devices with nanostructured bottom electrodes show light emission from the entire device area.

Furthermore, the antenna-coupled devices exhibit strongly enhanced light emission intensity, in particular in the spectral 
domain of the transmission peaks discussed previously. The electron-to-photon conversion efficiency is increased by two orders of magnitude from $Q E_{\mathrm{D} 0} \approx 4 \times 10^{-7}$ to $Q E_{\mathrm{D} 3} \approx 2.5 \times 10^{-5}$ at $V_{\mathrm{b}}=2.5 \mathrm{~V}$. A direct comparison of the transmission and emission spectra shows good agreement in spectral width and shape. The spatial non-uniformity of the emitted light intensity is due to fabrication-related contamination at the $\mathrm{Au} / \mathrm{h}-\mathrm{BN}$ interfaces and the roughness of the polycrystalline electrodes to which the atomically flat h-BN crystal cannot conform perfectly. Photon emission can be made spatially more uniform by using monocrystalline or template-stripped Au electrodes.

As reported by Lambe and McCarthy, inelastic electron tunnelling results in broadband light emission with a highfrequency cutoff given by

$\hbar \omega_{\max }=\left|e V_{\mathrm{b}}\right|$

where $\hbar \omega_{\max }$ is the photon energy, $e$ is the electron charge and $V_{\mathrm{b}}$ is the tunnel bias voltage. According to equation (1), the antenna-specific resonances should only be excitable if the applied bias exceeds the energy of the antenna mode. As shown in Fig. 3c, this behavior is indeed observed. The figure shows light emission spectra for device 3 as a function of applied bias $V_{\mathrm{b}}$. The experimental data show good agreement with equation (1).

To understand the role of the slot antennas in the light generation process, we studied the polarization and radiation characteristics of the emitted light. Figure 2a shows emission spectra for device 3 as a function of polarizer angle. The light intensity is maximized when the polarizer is oriented parallel to the short axis of the slots. For a polarizer angle parallel to the long axis of the slots, $>80 \%$ extinction is observed. Figure $2 \mathrm{~b}$ shows the photon momentum distribution in the back focal plane of the microscope objective (NA 1.4). Light is preferentially emitted in the $x$ direction, that is,
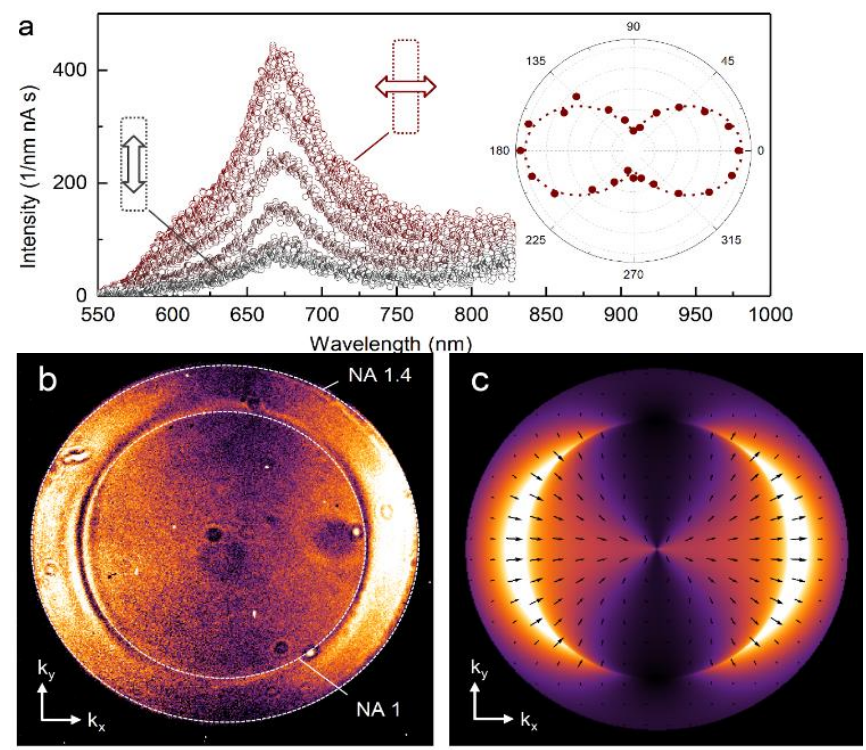

Figure 2: Light emission from the tunnel junction. (A) Polarization measurement of the emitted light, showing that the light is mostly polarized along the short axis of the slots. (B) A Fourier space image showing the angular distribution of the emitted photons. (C) Calculated angular spectrum of emission from a magnetic dipole oriented along the long axis of the slot.

parallel to the short axis of the slots. The measured polarization and the back focal plane intensity distribution qualitatively agree with the radiation generated by a magnetic dipole over a dielectric substrate ${ }^{40}$ and oriented along the long axis of the slots (Fig. 4c). These results are in agreement with the notion that slots can be viewed-in terms of Babinet's principle - as the complement of linear rod antennas, radiation from which is dominated by an electric dipole mode along the long axis of the $\operatorname{rod}^{41}$. However, as will be discussed later, slots also support electric dipole resonances parallel to their short axis. 
In a final experiment, we demonstrate ultrafast temporal modulation of light emission from h-BN tunnel junctions. The device is driven with an RF signal of frequency $f$, and a histogram of interphoton arrival times in the resulting photon stream is recorded by employing time-correlated single-photon counting (TCSPC). Figure 3A shows an excerpt from such a histogram recorded for a modulation frequency of $f=10 \mathrm{MHz}$, demonstrating that the photon stream is indeed time-modulated at the same frequency $f$. This can be seen unambiguously by averaging and Fourier-transforming the raw histogram. Following this approach, we modulated photon emission from the devices at frequencies ranging from $f=10$ $\mathrm{MHz}$ to $f=1 \mathrm{GHz}$, as shown in Fig. 3B-D.
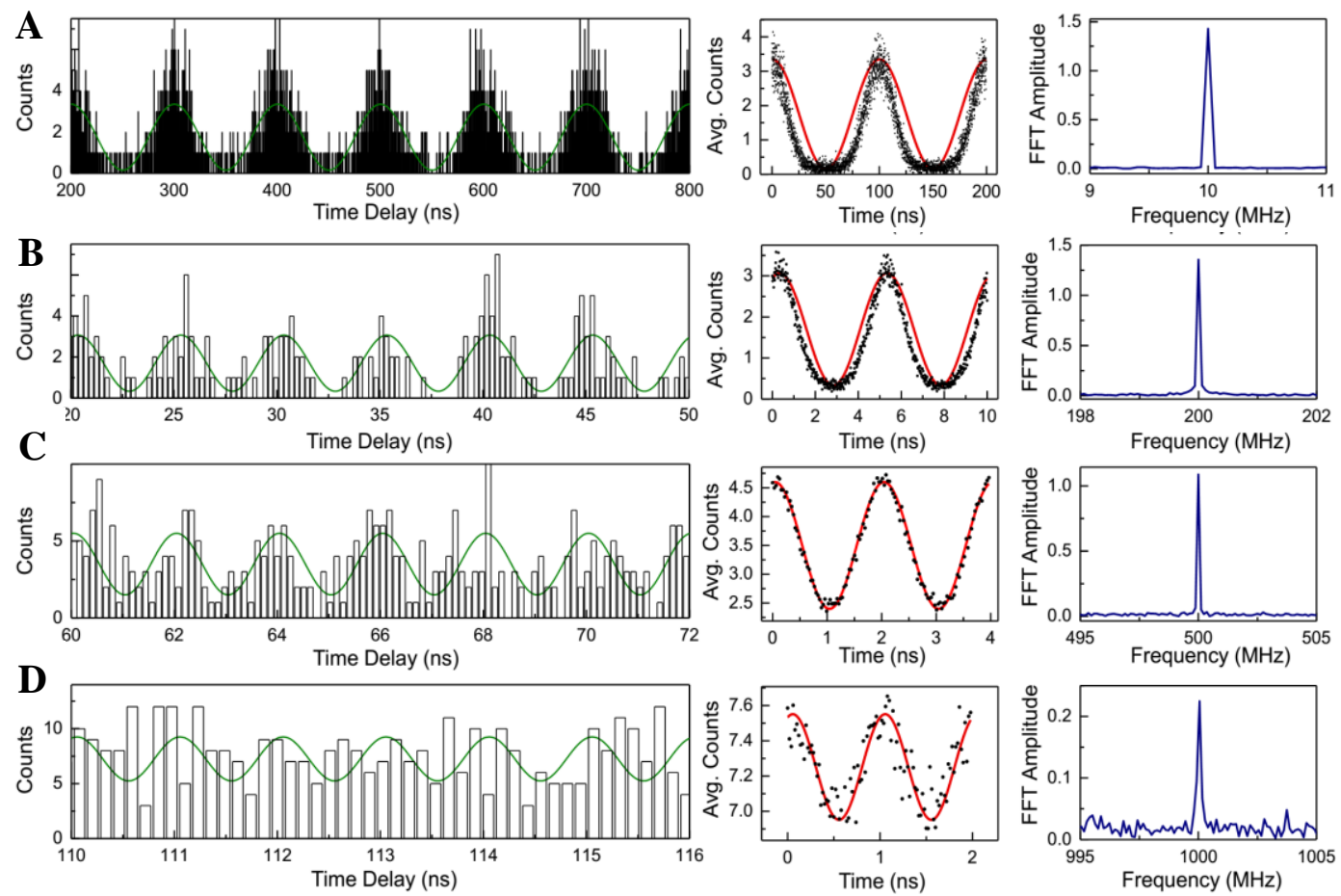

Figure 3: Modulation of light emission from the tunnel junction from $10 \mathrm{MHz}$ up to $1 \mathrm{GHz}$. (A)-(D) show the raw interphoton arrival time histograms as well as the averaged data, together with the Fourier transforms of the raw histograms.

As mentioned above, the speed of a tunnel light emitting device is fundamentally limited only by the tunneling times. However, in practice the RC time constant of the tunnel junction imposes an upper cut-off frequency. Based on our device dimensions and the thickness of the h-BN flake, we expect the capacitance of our junction to be of the order of $0.1 \mathrm{pF}$, implying a cutoff frequency of around $100 \mathrm{GHz}$. We could not reach this frequency in our measurements due to the timing jitter of the single photon counting avalanche photodiodes we used. It should be noted that our devices are rather large $\left(\sim 1 \mu \mathrm{m}^{2}\right)$ and with a reduction in the active junction area, the speed of the devices can reach the $\mathrm{THz}$ regime.

\subsection{Antenna-directed emission from $\mathrm{MoS}_{2}$ monolayers}

We also report on combining plasmonic antennas with 2D materials to achieve emission directionality from 2D monolayers. We demonstrate the coupling of optically excited excitons in a $\mathrm{MoS}_{2}$ monolayer to a plasmonic dimer antenna by precisely positioning the monolayer between two gold nanoparticles of the same size. To accomplish this, one of the particles was fixed under the $\mathrm{MoS}_{2}$ flake whereas the other one was mobile attached to a scanning probe for easy manipulation (Fig. 4A). The photoluminescence (PL) and angular distribution of photons as a function of the position of scanning top particle were recorded. The resulting spatial PL map is shown in Fig. 4B together with the simultaneously recorded topography. Maximal PL is seen when the two particles are aligned on top of each. This is 
despite the fact that the fields in the gap between the particles are oriented predominantly along $z$ and hence do not cause absorption in the $\mathrm{MoS}_{2}$. The increased PL is likely due to increased absorption by the fringing fields in the dimer antenna gap, as well as emission enhancement caused by coupling the low quantum yield $\mathrm{MoS}_{2}$ excitons to the antenna.

Interestingly, the angular distribution of PL photons is drastically modified by coupling to the dimer antenna. The inplane excitonic dipoles induce a strong out-of-plane secondary dipole in the dimer antenna, and as a result, the PL goes out at grazing angles (Fig. 5).

The coupling between excitons and the out-of-plane plasmonic mode can happen only if mirror symmetry about the $\mathrm{MoS}_{2}$ is somehow broken. In our experiment, this is the case because the $\mathrm{MoS}_{2}$ flake is much closer to one particle than the other.

A

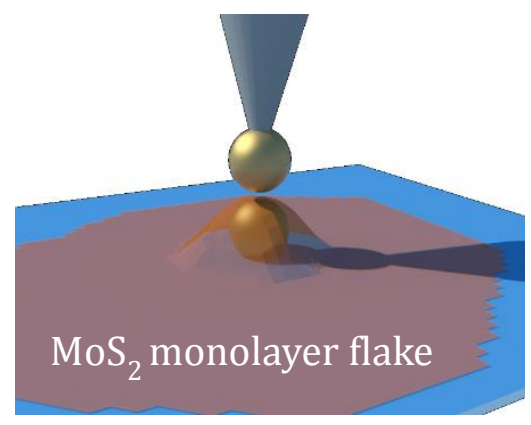

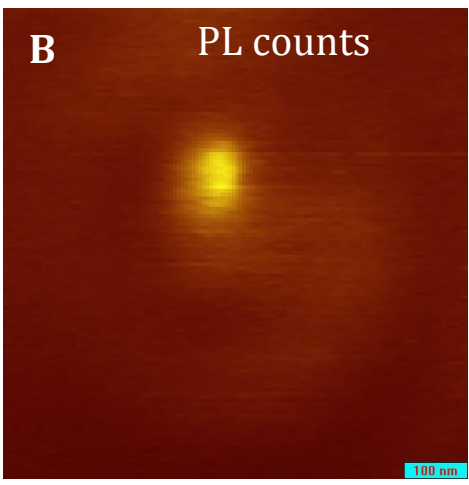

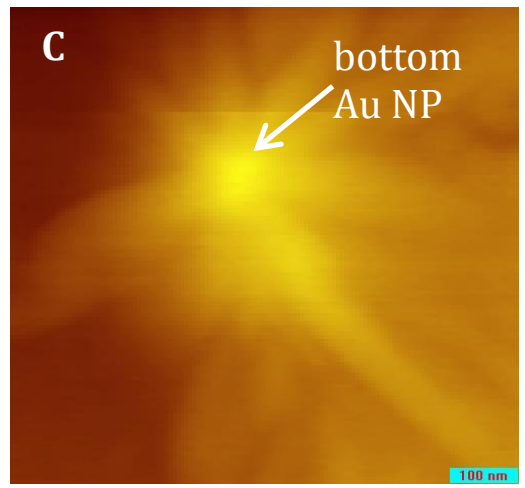

Figure 4: $\mathrm{MoS}_{2}$ coupled to a Au dimer antenna. (A) Schematic of the experiment. (B) PL recorded as the top particle is scanned over the $\mathrm{MoS}_{2}$ flake. (C) Topography image showing that the strongest PL occurs when the two particles are aligned on top of each other.

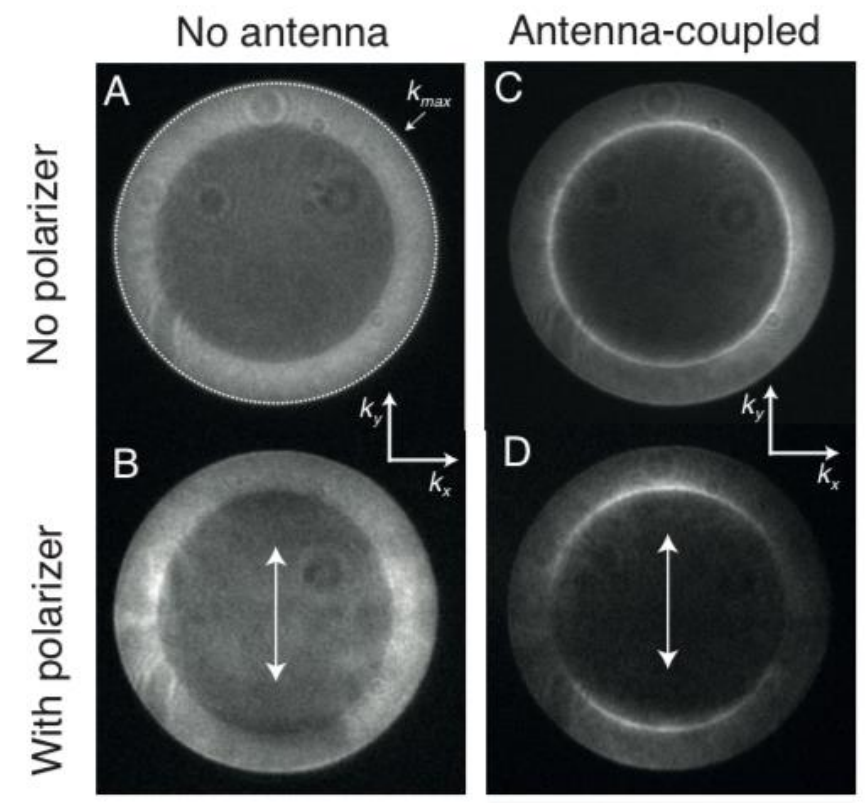

Figure 5: Fourier plane imaging of PL from $\mathrm{MoS}_{2}$ coupled to a dimer antenna. (A) and (B) Angular distribution from uncoupled bare $\mathrm{MoS}_{2}$. The inner ring corresponds to the critical angle for the glass-air interface $(\mathrm{NA}=1)$. (C) and (D) Angular distribution of PL from antenna-coupled $\mathrm{MoS}_{2}$. With a vertically oriented polarizer placed before the camera (D), the two lobes show that the emission is polarized as expected for a z-dipole. 


\section{REFERENCES}

[1] F. Withers et al, "Light-emitting diodes by band-structure engineering in van der Waals heterostructures", Nature Mater. 14, 301-306 (2015).

[2] N. Flöry et al, "A WSe $/ \mathrm{MoSe}_{2}$ heterostructure photovoltaic device”, App. Phys. Lett. 107, 123106 (2015).

[3] M. Parzefall et al, "Antenna-coupled photon emission from hexagonal boron nitride tunnel junctions", Nature Nanotech. 10, 1058-1063 (2015).

[4] P. Bharadwaj, A. Bouhelier, and L. Novotny, "Electrical excitation of surface plasmons", Phys Rev. Lett. 106, 226802 (2011).

[5] J. Kirtley, T. Theis, and J. Tsang, "Light emission from tunnel junctions on gratings", Phys. Rev. B 24, 5650 (1981).

[6] J. K. Gimzewski, B. Reihl, J. H. Coombs and R. R. Schlittler, "Photon emission with the scanning tunneling microscope", Z. Phys. B 72, 497-501 (1988).

[7] H. T. Miyazaki and Y. Kurokawa, "Squeezing visible light waves into a 3-nm-thick and 55-nm-long plasmon cavity", Phys. Rev. Lett. 96, 097401 (2006).

[8] X. Chen et al., "Atomic layer lithography of wafer-scale nanogap arrays for extreme confinement of electromagnetic waves", Nature Commun. 4, 2361 (2013). 